\title{
RESPOSTA A IMPACTO BALÍSTICO DE TECIDO DE ARAMIDA IMPREGNADO COM UM FLUIDO NÃO- NEWTONIANO *
}

André Ben-Hur da Silva Figueiredo ${ }^{1}$ Monique Vital de Lima² Eustáquio de Souza Baêta Junior ${ }^{3}$ Letícia dos Santos Aguilera ${ }^{4}$

\section{Resumo}

Foi avaliada a resposta a impacto balístico de um compósito constituído de tecido de aramida impregnado com um fluido não-Newtoniano para cinco concentrações de sílica $\left(\mathrm{SiO}_{2}\right)$ em polietileno glicol (PEG-200). A sílica foi caracterizada por difração de raios $X(D R X)$ e apresentou um tamanho médio de cristalito de $571 \mathrm{~nm}$. Os testes de impacto foram realizados em velocidade subsônica utilizando um sistema de lançamento de projétil de ar comprimido com uma pressão de 150 bar. Os resultados indicaram que o compósito com $67 \%$ em massa de $\mathrm{SiO}_{2}$ teve a menor profundidade de penetração em um anteparo de Medium Density Fiberboard (MDF).

Palavras-chave: Fluidos Não-Newtonianos; Blindagem Balística; Impacto Balístico; Fluidos Espessantes por Cisalhamento.

\section{RESPONSE TO BALLISTIC IMPACT OF ARAMID FABRIC IMPREGNATED WITH A NON-NEWTONIAN FLUID}

\begin{abstract}
The response to ballistic impact of a composite composed of aramid fabric impregnated with non-Newtonian fluid was evaluated for five concentrations of silica $\left(\mathrm{SiO}_{2}\right)$ in polyethylene glycol (PEG-200). The silica was characterized by X-ray diffraction (XRD) and exhibited a mean crystallite size of $571 \mathrm{~nm}$. The impact tests were carried out at subsonic speed using a 150 bar compressed air system. The results indicated that the composite with $67 \%$ by mass of $\mathrm{SiO}_{2}$ had the lowest depth of penetration in a Medium Density Fiberboard (MDF) bulkhead.
\end{abstract}

Keywords: N-Newtonian Fluids; Ballistic Shielding; Ballistic Impact; Shear Thickening Fluids

1 Bacharel em Física, Mestre em Engenharia Nuclear, Doutor em Ciência dos Materiais, Professor, Curso Básico e Seção de Engenharia Mecânica e de Materiais, Instituto Militar de Engenharia, Rio de Janeiro, RJ, Brasil.

2 Graduando em Engenharia de Fortificação e Construção, Instituto Militar de Engenharia, Rio de Janeiro, RJ, Brasil.

3 Bacharel em Engenharia Metalúrgica, Mestre em Ciência dos Materiais, Doutorando em Ciência dos Materiais, Seção de Engenharia Mecânica e de Materiais, Instituto Militar de Engenharia, Rio de Janeiro, RJ, Brasil.

4 Bacharel em Engenharia de Petróleo, Mestra em Ciência dos Materiais, Doutoranda em Ciência dos Materiais, Seção de Engenharia Mecânica e de Materiais, Instituto Militar de Engenharia, Rio de Janeiro, RJ, Brasil. 


\section{INTRODUÇÃO}

Desde tempos remotos, a humanidade busca o desenvolvimento de proteção corporal contra perigos e lesões. Esta necessidade levou à evolução de roupas protetoras denominadas armaduras. As armaduras são definidas como qualquer revestimento defensivo usado para proteger o corpo de ataques físicos. Peles de animais, fibras naturais e materiais metálicos como bronze, ferro ou aço foram usados para esta finalidade ao longo da história. Durante o século XX, as fibras naturais deram lugar às fibras sintéticas com as inovações na tecnologia de manufatura e na ciência dos materiais.

As armaduras modernas são projetadas para proteção contra projéteis, além da proteção contra perfuração e corte causados por objetos afiados. Elas em geral consistem em uma combinação de metal, cerâmicos e tecidos. Os tecidos são compostos de fibras poliméricas de alta resistência que proporcionam melhora na proteção e aumentam a mobilidade de soldados, policiais e pessoal de segurança $[1,2]$. Para melhorar a flexibilidade e reduzir o peso desses tecidos usando menos camadas e mantendo a mesma eficiência, foram desenvolvidas as armaduras líquidas. Tais armaduras são criadas impregnando os tecidos com fluidos espessantes por cisalhamento (FEC), que apresentam um comportamento nãoNewtoniano, ou seja, cuja viscosidade aparente aumenta com o aumento do estresse $[3,4,5]$. Esta característica os torna ideais para melhorar os materiais resistentes ao impacto, tais como equipamentos esportivos e de segurança.

O objetivo deste estudo é desenvolver FEC's baseado em sílica para aplicações em armaduras líquidas [6,7,8,9,10,11]. Para tal, foram produzidas misturas de partículas de sílica com PEG-200 usando cinco concentrações diferentes de $\mathrm{SiO}_{2}$. Essas misturas foram usadas para impregnar $49 \mathrm{~cm}^{2}$ de tecido de aramida e o desempenho do conjunto foi avaliado por meio de testes balísticos. Também foi realizada a caracterização por DRX e EDS do $\mathrm{SiO}_{2}$ empregado na produção dos FEC's.

\section{MATERIAIS E MÉTODOS}

Foram produzidos e caracterizados cinco compósitos, rotulados como A50, A60, A65, A67 e A68. O rótulo foi formado com a inicial do tecido de aramida (A), seguido pela concentração em massa de $\mathrm{SiO}_{2}$.

\subsection{Materiais utilizados}

Os materiais utilizados foram $\mathrm{SiO}_{2}$ em pó, com $98 \%$ de pureza, da marca Aldrich, PEG de $200 \mathrm{~g} / \mathrm{mol}, \mathrm{com} 98,5 \%$ de pureza, da marca Aldrich, etanol absoluto, com $98 \%$ de pureza, da marca Aldrich, e tecido de aramida bidirecional (AR - 0210) lisa de tela $X$, tratamento cru, com espessura de $0,28 \mathrm{~mm}$ e massa específica de $210 \mathrm{~g} / \mathrm{m}^{2}$. 


\subsection{Preparação das amostras}

Foram produzidas cinco misturas de $20 \mathrm{~g}$ de $\mathrm{SiO}_{2}$ e PEG-200 nas proporções em massa de $\mathrm{SiO}_{2}$ de $50 \%, 60 \%, 65 \%, 67 \%$ e 68\%, submetidas a ultrassom de 50 W e $40 \mathrm{kHz}$ por 30 minutos. Cada mistura foi diluída em $40 \mathrm{~mL}$ de etanol, submetida a ultrassom por 30 minutos e usada para impregnar $49 \mathrm{~cm}^{2}$ de tecido de aramida em vidro de relógio de $150 \mathrm{~mm}$ de diâmetro. Finalmente, o compósito foi seco em estufa a $79^{\circ} \mathrm{C}$ por $24 \mathrm{~h}$.

\subsection{Caracterização}

O tamanho médio de cristalito de $\mathrm{SiO}_{2}$ foi determinado a partir do difratograma obtido em um difratômetro de Raios $X$ Panalytical MPD, com radiação Cu-Ka (1,5406 Á) do Laboratório de Difração de Raios X do Instituto Militar de Engenharia (IME). Foi empregado para análise o software High Score Plus e o método de Scherrer que utiliza a largura a meia altura dos picos e a variação no ângulo de difração [12].

Para os ensaios balísticos foi usado um provete de ar comprimido da marca Gunpower modelo SSS, com dois cilindros comunicantes, um com capacidade de 0,5L e o outro de $6 \mathrm{~L}$, com pressão estimada em 150 bar e com projétil de chumbo calibre 22 de $3,3 \mathrm{~g}$. Para determinação da velocidade de boca e a energia em cada disparo, foi utilizado um cronógrafo balístico da marca Air Chrony modelo MK3, com precisão de 0,15 m/s, no Laboratório de Ensaios Balísticos (LEB) do IME.

\subsection{Ensaios balísticos}

Nos ensaios balísticos, o provete de ar comprimido foi posicionado a $5 \mathrm{~m}$ de distância do alvo, constituído por uma placa de MDF, como mostra a Figura 1. 0 provete (b) foi alinhado perpendicularmente ao alvo.

Placas de MDF (e) foram utilizadas como anteparos por se tratar de um material homogêneo, plano e denso, não possuindo os nós apresentados nas placas de madeira maciça.

Foi utilizado um supressor de ruídos (c) com a finalidade aumentar a estabilidade do projétil na saída do provete, diminuindo tanto o turbilhonamento provocado pela saída dos gases como também o ruído.

Para determinar a velocidade e a energia foi utilizado um cronógrafo balístico (d) situado a $10 \mathrm{~cm}$ da extremidade do supressor. 


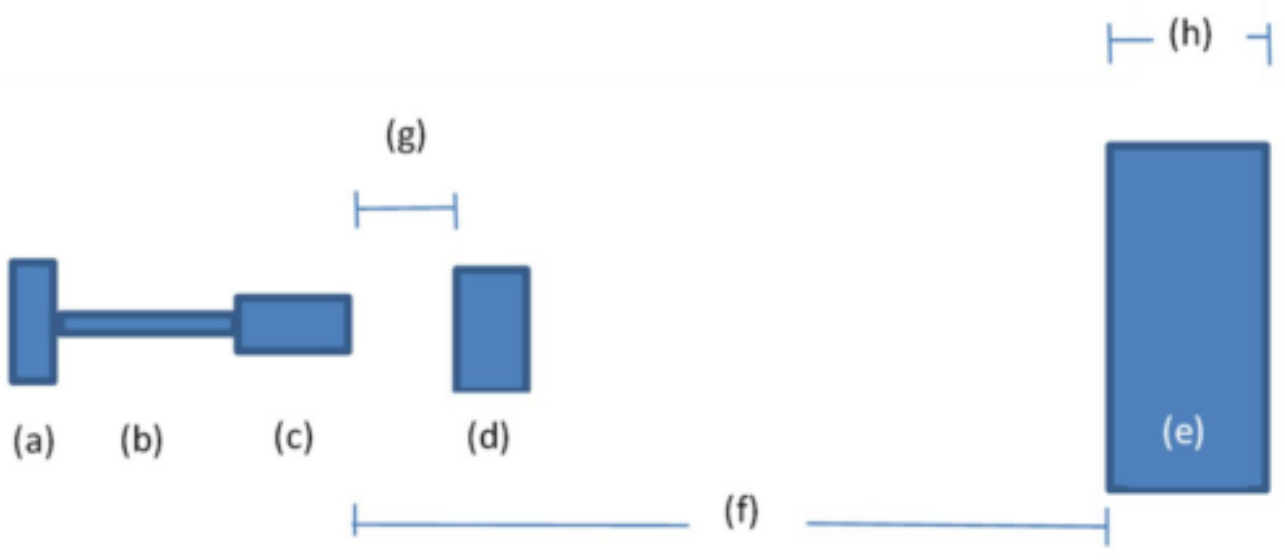

Figura 1 Representação esquemática do ensaio balístico com: (a) sistema de ar comprimido, (b) provete calibre 22, (c) supressor de ruídos, (d) cronógrafo balístico, (e) anteparo de MDF, (f) distância de $5 \mathrm{~m}$ entre a extremidade do supressor e o anteparo e (g) distância de $10 \mathrm{~cm}$ entre a extremidade do supressor e o cronógrafo.

A Figura 2 mostra a profundidade de penetração após cada disparo.

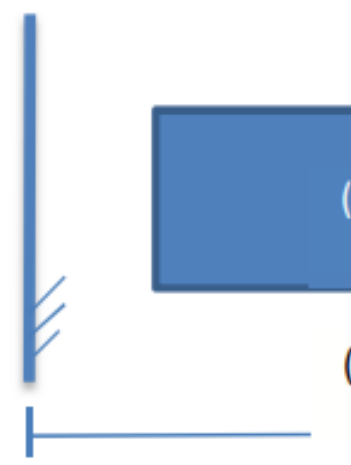

(b)

(a)

(c)

Figura 2 Representação esquemática da penetração do projétil no anteparo com: (a) a distância de penetração medida a partir da superfície do a anteparo até a ponta do projétil, (b) o projétil e (c) o MDF.

\section{RESULTADOS E DISCUSSÃO}

\subsection{Difratogramas de raios $\mathrm{X}$ do $\mathrm{SiO}_{2}$}

No difratograma do $\mathrm{SiO}_{2}$, mostrado na Figura 3, são observados os picos característicos da estrutura do quartzo [11], indexados através da ficha 96-710-3015 do banco de dados COD (Crystallography Open Database). Pelo método de Scherrer, o $\mathrm{SiO}_{2}$ pode ser considerado micrométrico, uma vez que o tamanho médio do cristalito é de $571 \mathrm{~nm}$. 


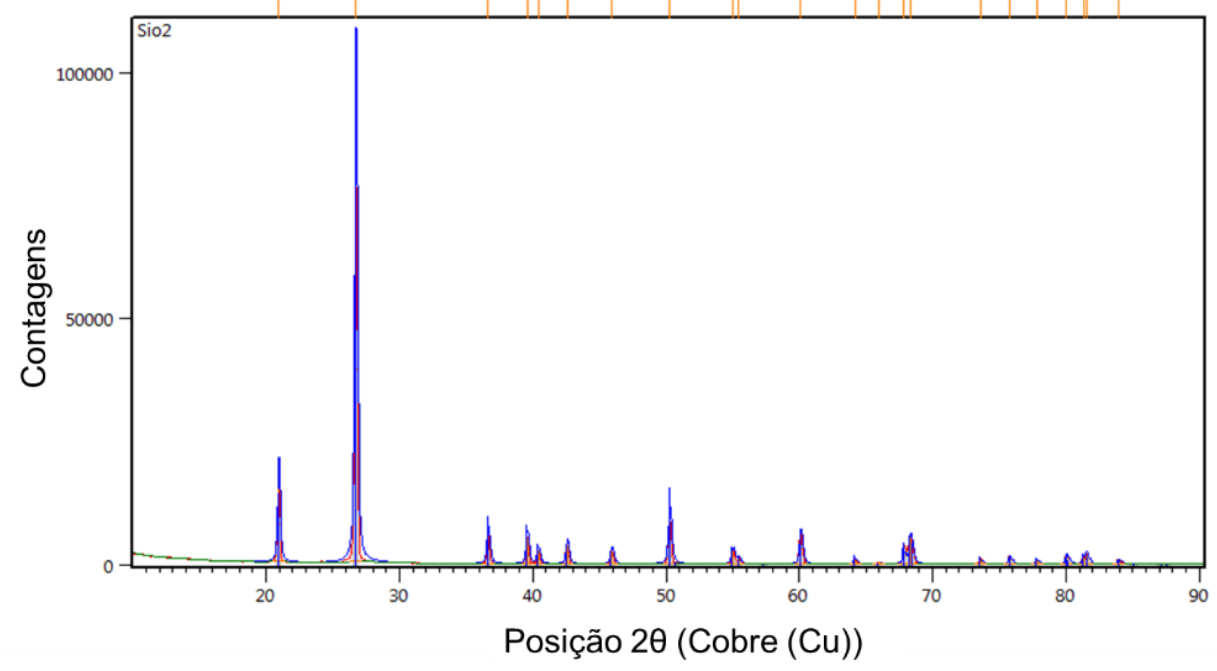

Figura 3 Difratograma de raios $\mathrm{X}$ da amostra de $\mathrm{SiO}_{2}$.

\subsection{Energy Dispersion Spectroscopy (EDS) do $\mathrm{SiO}_{2}$}

Como se pode ver na Figura 4, o EDS do $\mathrm{SiO}_{2}$ mostrou que, além de silício e oxigênio, o material contém traços de carbono. Também foi observada a presença de platina, utilizada no recobrimento da amostra.

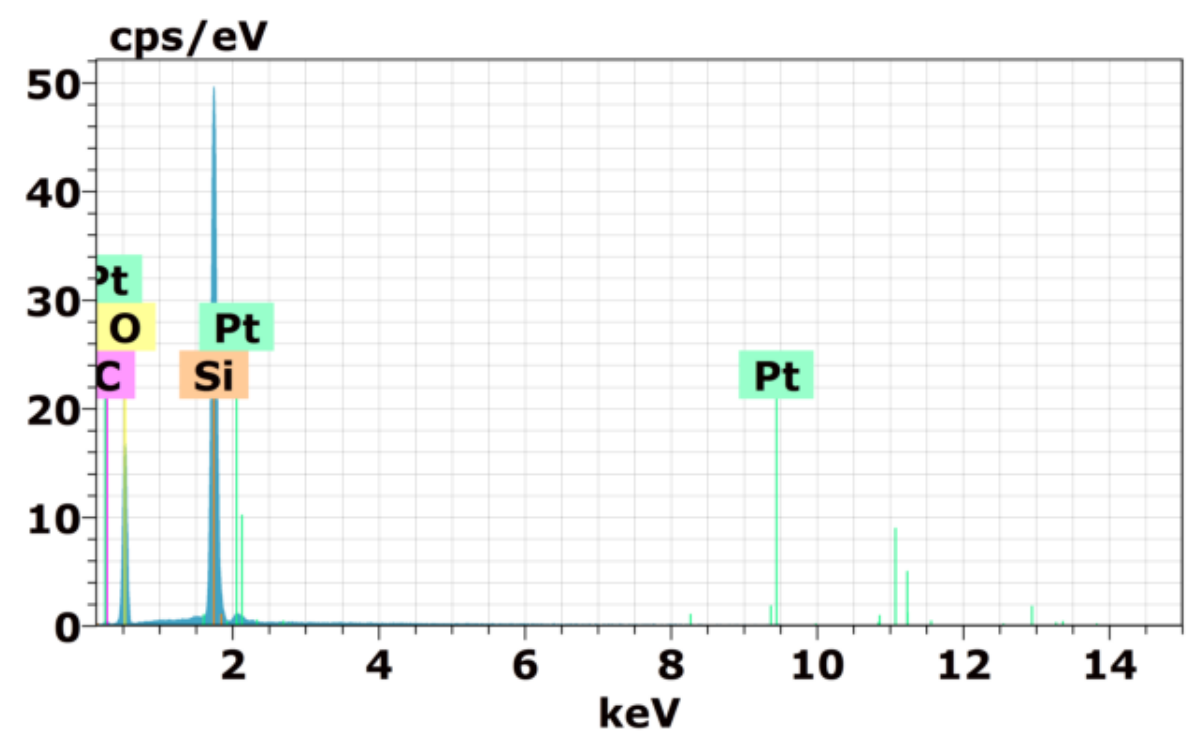

Figura 4 EDS do $\mathrm{SiO}_{2}$

\subsection{Ensaios balísticos}

Todos os disparos atravessaram os compósitos, como mostrado na Figura 5 , para as concentrações de $67 \%$ e $68 \%$. Foi realizado o mínimo de três disparos para cada compósito. 

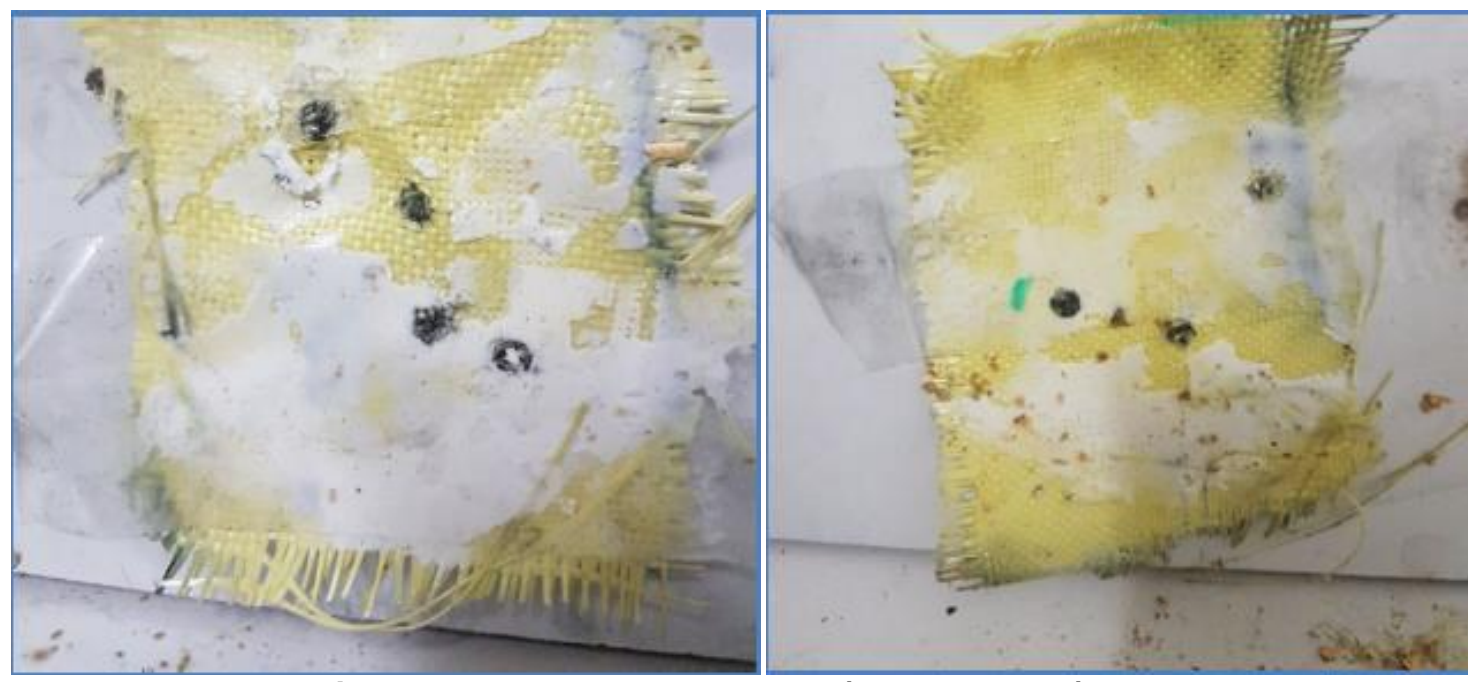

Figura 5: Amostras A67 e A68 após o ensaio balístico.

A Tabela 1 apresenta os compósitos com as respectivas velocidades médias de lançamento, a energia do projétil lançado e a profundidade média de penetração. Também foram relacionados os disparos efetuados no MDF, sem amostra (SA), e na aramida pura, sem adição de FEC. É possível verificar que as profundidades de penetração dos compósitos foram menores que o sem amostra (SA) e com aramida pura.

Tabela 1 Valores médios de velocidade, energia e profundidade de penetração, obtidos no ensaio balístico.

\begin{tabular}{|c|c|c|c|}
\hline COMPÓSITO & $\begin{array}{c}\text { Velocidade Média de Boca } \\
(\mathrm{m} / \mathbf{s})\end{array}$ & $\begin{array}{c}\text { Energia de Boca } \\
(\mathbf{J})\end{array}$ & $\begin{array}{c}\text { Profundidade de Penetração } \\
(\mathbf{m m})\end{array}$ \\
\hline SA & 212,49 & 74,51 & 27,85 \\
\hline ARAMIDA & 214,80 & 76,14 & 25,43 \\
\hline A50 & 211,83 & 74,03 & 16,35 \\
\hline A60 & 210,28 & 72,95 & 17,00 \\
\hline A65 & 217,02 & 77,71 & 16,40 \\
\hline A67 & 207,86 & 71,29 & 09,13 \\
\hline A68 & 211,52 & 73,83 & 15,57 \\
\hline
\end{tabular}

A Figura 6 mostra a profundidade média de penetração do projétil no anteparo sem amostra, com tecido de aramida pura e para cada compósito. A partir dela é possível observar que os cinco compósitos apresentaram melhor desempenho, ou seja, aquele para o qual a profundidade média de penetração foi menor, em comparação com a aramida pura. O melhor desempenho dos compósitos pode ser devido ao aumento na força de pullout nos fios do tecido de aramida após a transição do fluido espessante por cisalhamento para o estado de alta rigidez [13]. Também é possível observar que o melhor desempenho entre os compósitos é atribuído a A67, sugerindo ser a melhor composição entre $\mathrm{SiO}_{2}$ e PEG-200. Por fim, o tecido de aramida pura utilizado não teve efeito na redução da profundidade de penetração. 


\section{Profundidade de penetração}

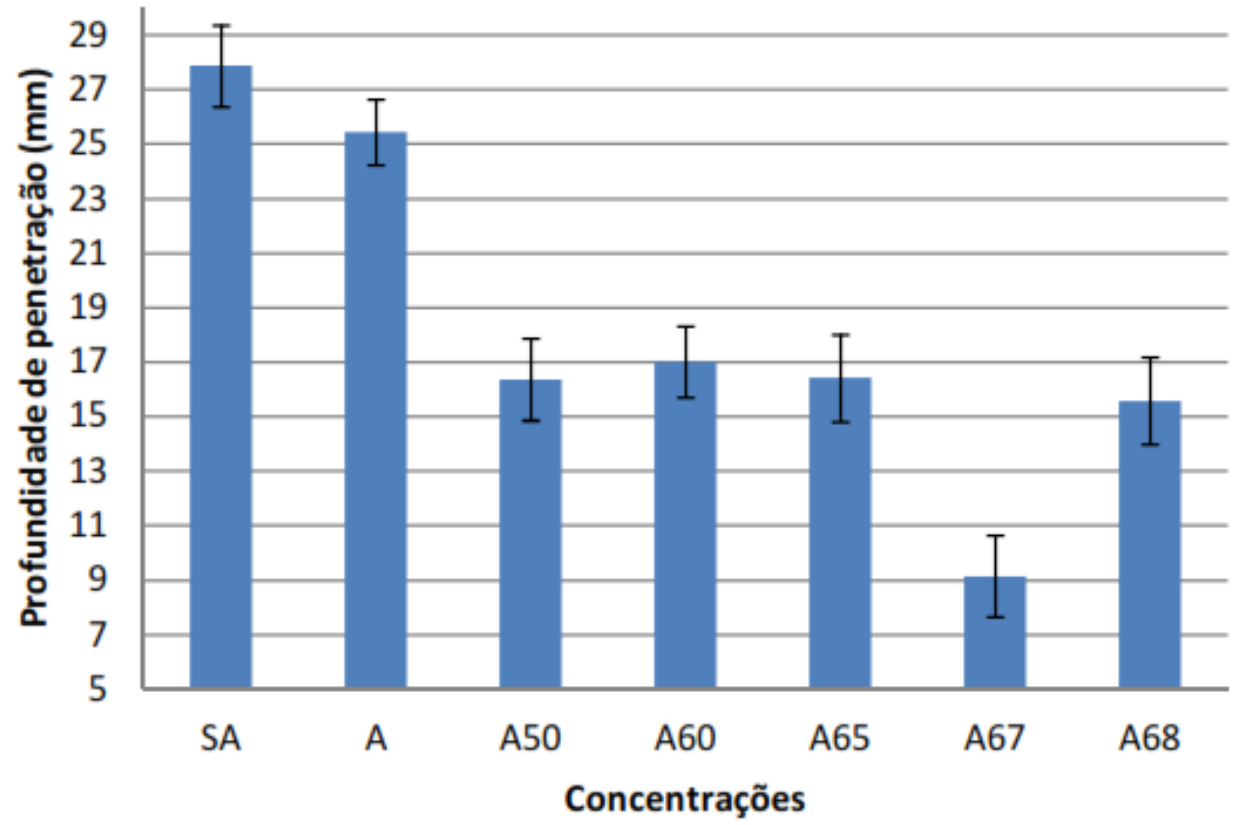

Figura 6 Profundidade de penetração no anteparo para cada compósito.

\section{CONCLUSÃo}

A caracterização por DRX da sílica empregada na produção das amostras mostrou que o material apresentava $100 \%$ da fase quartzo e um tamanho médio de cristalito de $571 \mathrm{~nm}$.

Os cinco compósitos apresentaram melhor desempenho em comparação com aramida pura, devido ao aumento na força de pullout nos fios do tecido de aramida após a transição do FEC para o estado de alta rigidez.

O compósito A67 foi o que apresentou o melhor desempenho entre os compósitos, sugerindo ser a melhor composição entre $\mathrm{SiO}_{2}$ e PEG-200.

\section{Agradecimentos}

Os autores agradecem ao Prof. Ronaldo Sergio de Biasi pela leitura cuidadosa do artigo. Agradecem também à CAPES e ao CNPq pelo apoio financeiro.

\section{REFERÊNCIAS}

[1] CAVALLARO, P.V., Soft body armor: an overview of materials, manufacturing, testing, and ballistic impact dynamics, 1 august 2011, NUWC-NPT Technical Report 12,057

[2] EGRES, R.G. Jr., LEE, Y.S., KIRKWOOD, J.E., KIRKWOO, K.M., WETZEL E.D. , WAGNER N.J., Liquid armor: Protective fabrics utilizing shear thickening fluids, 
October 26-27, 2004 IFAI $4^{\text {th }}$ Int. Conf. on Safety and Protective Fabrics, Pittsburgh, PA.

[3] BARNES, H. A., HUTTON, J.F., WALTERS, K., An introduction to rheology, 1989, Rheology Series 3, Elsevier.

[4] SUN, L. -L., XIONG, D. -S., XU, C.-Y., Application of shear thickening fluid in ultra high molecular weight polyethylene fabric, J.Appl. Polym. Sci, v. 129, p. 1922-1928, 2013.

[5] MAJUMdAR, A., BUTOLA, B. S., SRIVASTAVA, A., Development of soft composite materials with improved impact resistance using Kevlar fabric and nano silica based shear thickening, Materials and Design, v. 54, p. 295-300, 2014.

[6] WU, Q.-M., RUAN, J.-M., HUANG, B.-Y., ZHOU, Z.C., ZOU J.-P., Rheological behavior of fumed silica suspension in polyethylene glycol, J. Cent. South Univ. Technol., v. 13, p. 1, 2006.

[7] GÜRGEN, S., LI, W., KUSHAN, M.C., The rheology of shear thickening fluids with various ceramic particle additives, Mater. Design, v. 104, p. 312-319,2016.

[8] DECKER, M.J., HALBACH, C.J., NAM, C.H., WAGNER, N.J., WETZEL, E.D., Stab resistance of shear thickening fluid (STF)-treated fabrics, Comp. Scs. Tech., v. 67, p. 565-578, 2007.

[9] EGRES, R.G., DECKER, M.J., HALBACH, C.J., LEE, Y.S., KIRKWOOD, J.E., KIRKWOOD, K.M., WAGNER, N.J. Stab resistance of shear thickening fluid(STF) Kevlar composites for body armor applications, Proceeding of the $24^{\text {th }}$ Army Science Conference, Orlando, FL. Nov. 29 - Dec. 2, 2004.

[10] DING, J., TRACEY, P.J., LI, W., PENG, G., WHITTEN, P.G. Review on shear thickening fluids and applications, Textiles and Light Industrial Science and Technology, v. 2, Issue 4, October 2013.

[11] VARELA, M.L., NASCIMENTO, R.M., MARTINELLI, A.E., HOTZA, D., MELO, D.M.A., MELO, M.A.F. Otimização de uma metodologia para análise mineralógica racional de argilominerais (Otimization of rational mineralogical analysis of ceramics), Ceramica, v. 51, p. 387-397, 2005.

[12] SURYANARAYANA, C., NORTON, M.G. X-Ray Diffraction - A Practical Approach. New York: Plenum press, 1988.

[13] LEE, Y.S., WETZEL, E.D., WAGNER, N.J., The ballistic impact characteristics of Kevlar woven fabrics impregnated with a colloidal shear thickening fluid, J. Mat. Scs., v. 38, p. 2825-2833, 2003. 